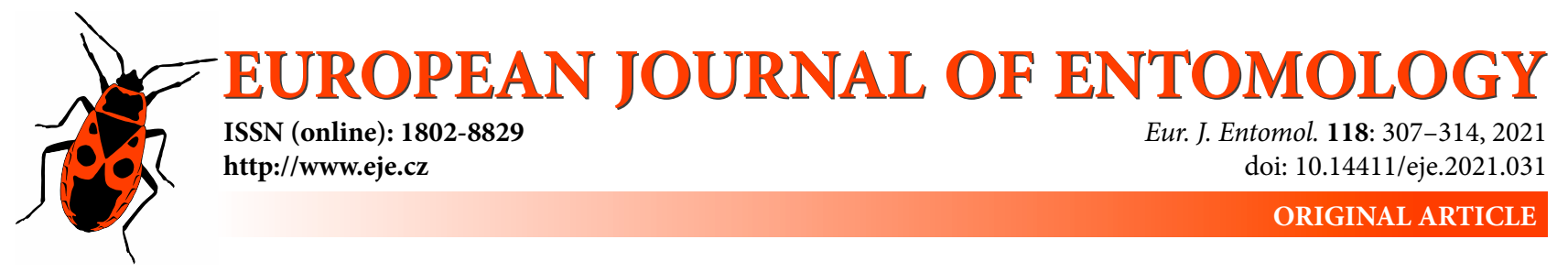

\title{
Relationship between Rab and insulin-like proteins in the nervous system of Bombyx mori
}

\author{
Tomohide UNO ${ }^{1}$, Yusuke OZAKIYA ${ }^{1}$, MaKo SASAO $^{1}, \mathrm{Katsuhiko} \mathrm{SAKAMOTO}^{1}$, Yasuo YAMAUChI $^{2}$, YUICH UNO $^{3}$, \\ Kengo KANAMARU ${ }^{1}$ and AKIRA MIZOGUCHI ${ }^{4}$ \\ ${ }^{1}$ Department of Applied Chemistry in Bioscience Biological Chemistry, Faculty of Agriculture, Kobe University, Nada-ku, Kobe \\ Hyogo 657-8501, Japan; e-mails: unotom@kobe-u.ac.jp, ksakamoto@diamond.kobe-u.ac.jp, kng@kobe-u.ac.jp \\ ${ }^{2}$ Graduate School of Agricultural Science, Kobe University, 1-1 Rokkodai-cho, Nada-ku, Kobe, Hyogo 657-8501, Japan; \\ e-mail: yamauchi@kobe-u.ac.jp \\ ${ }^{3}$ Department of Plant Resource Science, Faculty of Agriculture, Kobe University, Nada-ku, Kobe, Hyogo 657-8501, Japan; \\ e-mail: yuno@kobe-u.ac.jp \\ ${ }^{4}$ Division of Liberal Arts and Sciences, Aichi Gakuin University, Nisshin, Aichi 470-0195, Japan; e-mail: amizo@dpc.agu.ac.jp
}

Key words. Lepidoptera, Bombyx mori, larva, Rab proteins, bombyxin, brain, corpus allatum

\begin{abstract}
Rab proteins are small GTP-binding proteins and are the largest family in the Ras GTPase superfamily and mediate vesicular transport in cells. Diverse insulin-like peptides, such as bombyxin, are synthesized in the brain and secreted into the haemolymph by the corpus allatum (CA). In the brain of Bombyx mori, Rabs are expressed in a specific area; however, which Rabs actually link the secretion of bombyxin remains unknown. A double-staining analysis of nine Rabs (Rab1, 3, 6, 7, 14, 21, 26, 39 and X4) and bombyxin indicated that Rab3-, Rab7-, Rab39- and RabX4-immunohistochemical reactivity (ir) areas overlapped with bombyxin-ir in the brain and CA in B. mori, while Rab6-, Rab14- and Rab21-irs partially overlapped in the CA. Rab1-ir occurred in the other immunopositive areas in CA. Rab26-ir did not occur in the brain. Rab39-ir occurred in UNC104, Rab39- effector, -immunopositive neurons in the brain and CA. Thus, Rab3, 7, 39 and X4 may regulate the exocytosis of bombyxin.
\end{abstract}

\section{INTRODUCTION}

Rab proteins belong to a large family of small GTPases that direct intracellular vesicular trafficking (Barr, 2013; Li \& Marlin, 2015; Pfeffer, 2017). About 70 Rab GTPases are encoded in the human genome, up to 31 in Drosophila melanogaster and 11 in yeast (Brighouse et al., 2010). Each Rab specifically targets a distinct membrane compartment, e.g., Rab1 the transport of vesicles in the endoplasmic reticulum (ER) to the Golgi apparatus, Rab3 to the secretory vesicle, Rab6 and Rab39 to the Golgi apparatus, Rab14 and Rab21 to early endosome, Rab26 to secretory granules, Rab7 to late endosomes. Active GTP-bound Rab proteins, recruit their cognate Rab effectors to the membrane surfaces, and, thereby, cooperate with the effectors to mediate diverse processes in intracellular membrane trafficking, including vesicle formation, vesicular transport, membrane tethering and fusion to target compartments (Pfeffer, 2013). For example, UNC104 interacted with Rab39 and is a kinesin-related protein (ATPase, the Drosophila ortholog of the kinesin-3 KIF1A), which is a motor protein (Gillingham et al., 2014). UNC104 is required for transport of ILPs along the axons of insulin-producing cells (Cao et al.,
2014). In insects, Rab proteins participate in important cellular processes, such as the immune response, autophagy, neurotransmitter release, phagocytosis, development, fertility and oogenesis (Lighthouse et al., 2008; Uytterhoeven et al., 2011; Ye et al., 2012; Garg \& Wu, 2014; Fujita et al., 2017; Elmogy et al., 2018; Caviglia et al., 2019).

Insect neuropeptides regulate insect-specific phenomena, such as development, ecdysis, feeding, metamorphosis and moulting. These neuropeptides are mainly synthesized in the brain, transported via axons and finally secreted through a neurosecretory organ such as corpus allatum (CA) into the haemolymph (Nassel, 2002; Heuer et al., 2012; Schoofs et al., 2017). In vertebrates, insulin and insulin-like growth factors are well known as key regulators of metabolism, development and growth and belong to a superfamily of structurally related proteins (Claeys et al., 2002; White, 2002). In D. melanogaster, many biological events, such as body size, metabolism, lifespan and reproduction, are controlled by insulin-like peptides (ILPs), in particular DILP1-7 (Nassel et al., 2015; Nassel \& Vanden Broeck, 2016). 
Bombyxin is a $5 \mathrm{kDa}$ peptide secreted by the brain an an ILP found in Lepidoptera (e.g., butterflies and moths) and comprises highly heterogeneous molecular forms (Nagasawa et al., 1986; Van de Velde et al., 2007). Bombyxin monoclonal antibody immune stains four pairs of large mid-dorsal neurosecretory cells in the brain and thick and dense nerve fibres around the periphery of the CA (Mizoguchi \& Okamoto, 2013). Bombyxin regulates trehalose and glycogen metabolism (Satake et al., 1997), induces meiosis in ovaries and its signalling is linked to nutrition in Bombyx mori (Nagata et al., 2008; Mizoguchi \& Okamoto, 2013).

In mammals, Rab proteins participate in the insulin secretory vesicle fusion process. Rab3A and Rab27A have important roles in insulin-containing granule docking and/or priming of pancreatic $\beta$-cells (Wang \& Thurmond, 2009). Rab3A-I- mice are glucose intolerant, which could result from a significant loss in glucose-stimulated insulin release. Similarly, Rab27A's depletion decreased the exocytosis that is triggered by a cocktail containing glucose. A Rab2A knockdown inhibits insulin secretion (Sugawara et al., 2014). Little is known about how insect Rab mediates the exocytosis of ILPs

To clarify the relationship between some Rabs and neuropeptide secretion, we carried out an immunohistochemical analyses of the brain of $B$. mori using antibodies against Rabs. Rab1, 3, 6, 7 and 14 co-localize with bombyxin in the brain of B. mori (Uno et al., 2013, 2016). Furthermore, Rab3 and Rab6 co-localize with bombyxin in the CA. There are many Rabs, which are co-localized with bombyxin-immunohistochemical reactivity (ir), in the brain of B. mori, but it is unknown which Rab proteins function in bombyxin secretion.

Therefore, the present study aimed to investigate the relationships between nine Rabs and bombyxin secretion in the nervous system of $B$. mori. In this study four new antibodies for Rabs and Rab39-specific effector were used to identify Rab-expressing cells, locate their regional distribution and determine whether they co-localize with bombyxin in the brain and CA.

\section{MATERIALS AND METHODS}

\section{Materials and insect cultures}

Oligonucleotides were purchased from Invitrogen (Tokyo, Japan). The other chemicals used were of the purest grade commercially available. We used hybrids (Kinshu $\times$ Showa or Daizo, p50 strains) of B. mori. Day 5 fourth-instar larvae were used in all of the experiments.

\section{Purification of B. mori UNC 104, Rab21, 26 and 39, and the production of antiserum}

The cDNA fragments containing the coding sequences of $B$. mori Rab21 (NCBI Reference Sequence. XM_038011422), Rab26 (NCBI Reference Sequence. XM_004923206) and Rab39 (NCBI Reference Sequence XM_004930649) were generated by reverse transcriptase-PCR (RT-PCR) using oligonucleotides and then separately sub-cloned, and their sequences analysed using a DNA Sequencer. The partial cDNA fragment of the carboxy terminal region of UNC104 (NCBI Reference Sequence XM_021348078), which interacted with Rab39, was isolated and analysed. Primers used for Rab21, Rab26, Rab39 and UNC104 are 5'-GGATCCATGACTACTACGACTGGAGGTGC-3',

5'-TTAGGAACTCCTGTGTCCTGAACAGC-3',

5'-GGATCC ATGTGGAATCCGAATATGGATAAC-3',

5'-TAAACTTATGTACATGGCGGACACGATG-3',

5'-GGATCCATGGTCGATCCGATATTTGATTAT-3',

5'-CTAACAGCACGTTGAGTGTACAGCTTCTGCT-3',

5'-GGATCCTACATCACGCTCAGCGCTTACCTC-3',

5'-TCACGGGTTGATCGCGTACAGCCAGTCGTG-3' .

The fragment was inserted into expression vectors [pColdII (Takara Shuzo, Kyoto, Japan ) or pGEX6P-2 (GE Healthcare UK Ltd, UK)]. The expression of B. mori Rab proteins and UNC104 in E. coli (BL21 strain) and their purification were performed as previously described for other B. mori Rab proteins (Uno et al., 2014). The carboxy terminal region of UNC104 was used as an antigen.

Antisera to Rabs and UNC104 of B. mori were generated in a rabbit or rat. The sera were isolated and tested for the presence of anti- $B$. mori Rab antibodies using western immunoblotting. Antisera against Rab1, 3, 6, 7, 14, X4 and bombyxin of $B$. mori, and the GST- $B$. mori Rab proteins, were obtained as previously described (Uno et al., 2007).

\section{Western immunoblotting and immunohistochemistry}

Western blotting and immunohistochemistry were done as described previously (Uno et al., 2019). In western blotting, the membrane was incubated with the appropriate primary antibody as follows: anti-Rab serum $(1: 2,000)$. The membrane was then washed, followed by incubation with the secondary antibody, peroxidase-conjugated goat anti-rabbit $\operatorname{IgG}(1: 2,000)$, (Fuji-Film Wako chemicals, Miyazaki, Japan). After washing, proteins were detected using the peroxidase-staining DAB kit. (Nakalai Tesque Inc, Kyoto, Japan).

Insect heads or $15-12$ brains were fixed $\left(24 \mathrm{~h}\right.$ at $\left.4^{\circ} \mathrm{C}\right)$ in Bouin fluid (saturated picric acid, formalin and acetic acid at $15: 5: 1$ by volume). Standard histochemical methods were used for tissue dehydration (ethanol $80-100 \%$ and xylene), embedding in paraplast, sectioning (8- $\mu \mathrm{m}$-thick sections), deparaffinization (xylene) and rehydration (ethanol $70-100 \%$ ).

After blocking, sections of the insect heads were incubated in phosphate buffer with the primary antibodies, anti-Rab rabbit serum $(1: 500)$, anti-UNC104 rat serum $(1: 500)$ and anti-bombyxin mouse IgG $(1: 500)$. After washing, the sections were incubated with $7.5 \mu \mathrm{g} / \mathrm{ml}$ of a secondary antibody, donkey anti-mouse or anti-rat $\operatorname{IgG}(\mathrm{H}+\mathrm{L})-\mathrm{CF} 555$ and goat anti-rabbit $\operatorname{IgG}(\mathrm{H}+\mathrm{L})$ CF488 (Biotium Inc. Hayward, CA, USA). After washing and mounting, the sections were examined using a BX50 microscope (Olympus Corp., Japan) equipped with BX-FLA reflected light fluorescence and WIG and NIBA mirror/filter units. Three to five individuals were used in each immunocytochemical experiment. The excitation wavelength and emission range in WIG mirror/ filter units were $520-550 \mathrm{~nm}$ and above $580 \mathrm{~nm}$, respectively. In control experiments, the primary antibodies were replaced with the pre-immune rabbit serum. No significant staining was observed above the background level. Further the bleed-through of fluorescence was checked by imaging single stained samples through both filter/mirror units (WIG and NIBA). In both cases no significant staining was observed. Fig. 6 presents the percentage of immunopositive cells in sections of CA.

\section{RESULTS}

\section{Antibodies against Rabs of $B$. mori}

Antibodies produced against Rab21, 26 and 39 of B. mori specifically recognized the protein band corresponding to 
$\begin{array}{llll}\text { Antigen; } & \text { GST- } & \text { GST- } & \text { GST- } \\ & \text { BRab21 } & \text { BRab26 } & \text { BRab39 }\end{array}$

Antibody;anti-Rab21

$29 \mathrm{kDa}$

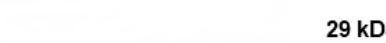

$29 \mathrm{kDa}$
$29 \mathrm{kDa}$

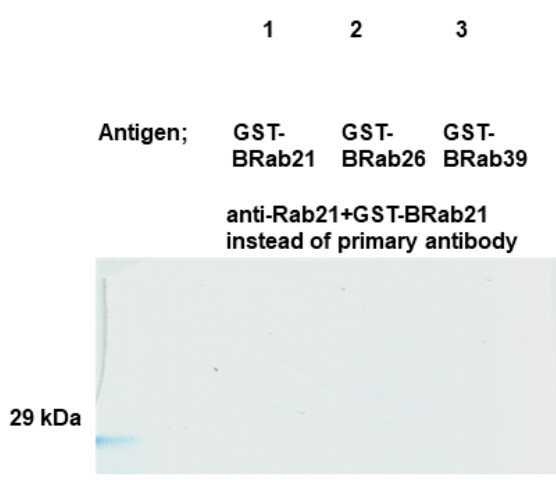

$\begin{array}{lll}\text { GST- } & \text { GST- } & \text { GST- } \\ \text { BRab21 } & \text { BRab26 } & \text { BRab39 }\end{array}$

Antibody;anti-Rab26

Antigen; GST- GST- GST-

BRab21 BRab26 BRab39

Antibody;anti-Rab39

11

12

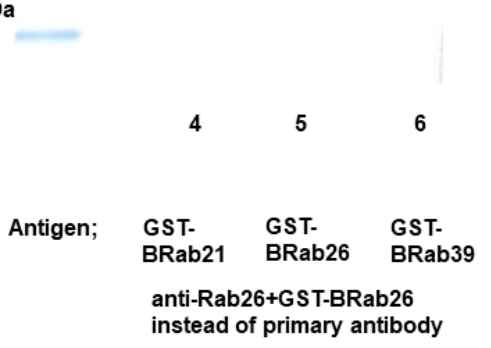

$29 \mathrm{kDa}$
$29 \mathrm{kDa}$

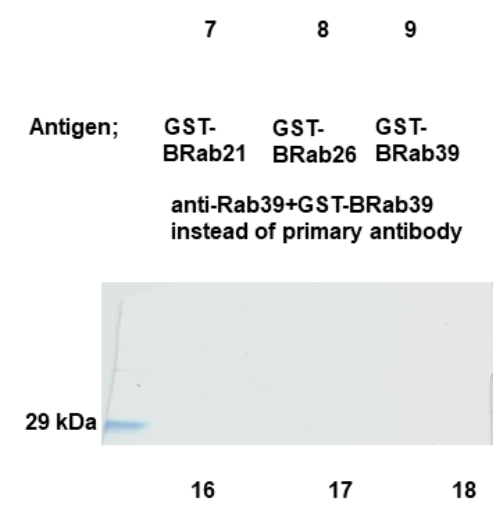

Fig. 1. Immunoblot analysis of the anti-Rab antibodies (Rab21, 26 and 39). Lane 1, 4, 7, 10,13 and 16, GST-BRab21. Lane 2, 5, 8, 11,14 and 17, GST-BRab26. Lane 3, 6, 9, 12,15 and 18, GST-BRab39. Lane1-3, anti-BRab21 antibody staining. Lane 4-6, anti-BRab26 antibody staining. Lane7-9, anti-BRab39 antibody staining. Lane 10-18, antibody-positive antigen used as the primary antibody (control). Expected sizes of fusion proteins were $45-46 \mathrm{kDa}$.

the position of the purified partial Rab of B. mori (Fig. 1, Lanes 1, 5 and 9). Control experiments (the addition of the antigen and primary antibody together) did not detect staining above background levels (Fig. 1, Lanes 10-18).

\section{Rab is present in specific neurons in the brain of B. mori}

Atnti-Rab39 and anti-Rab3 detected a restricted area in a set of neurons in the pars intercerebralis (PI), (Fig. 2d and 3j). Anti-Rab1, -Rab6, -Rab7, -Rab14, -Rab21 and
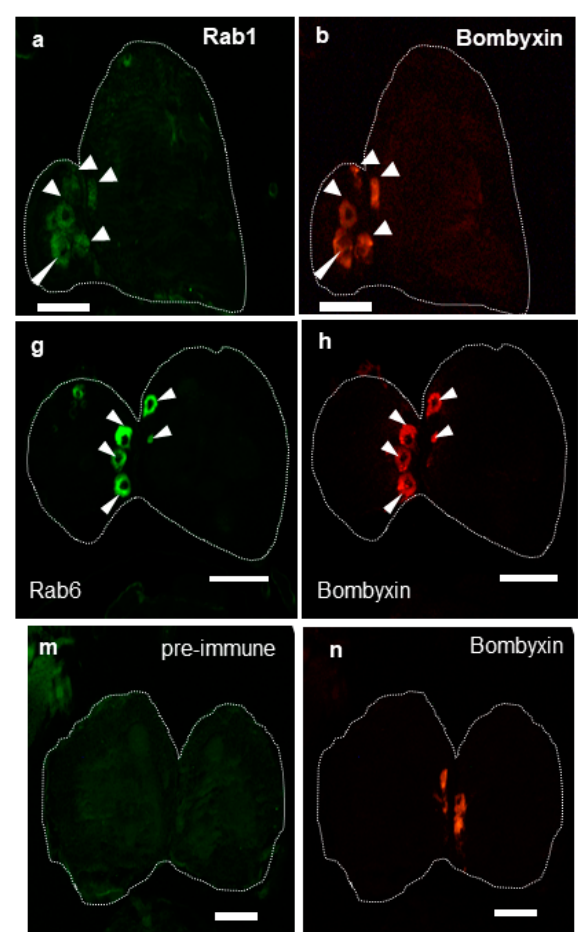
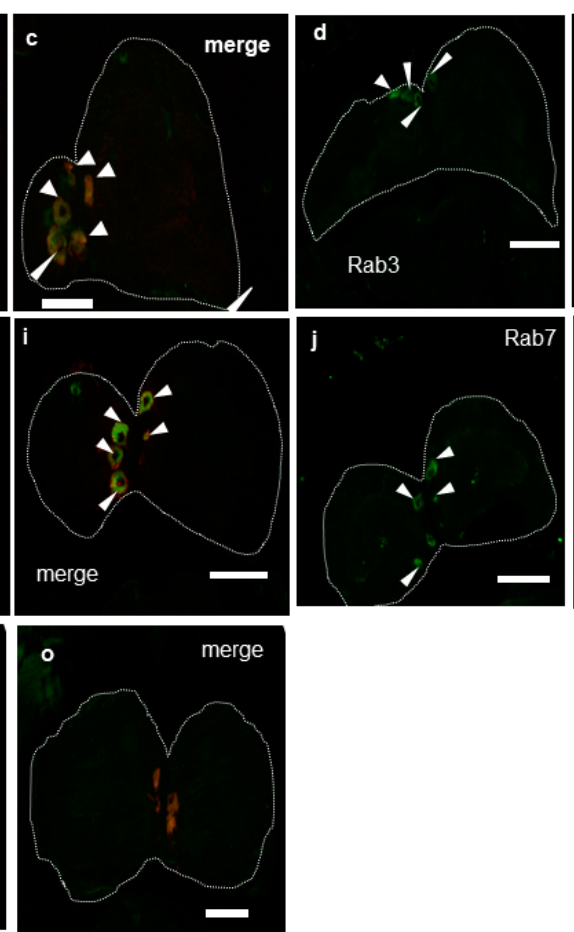
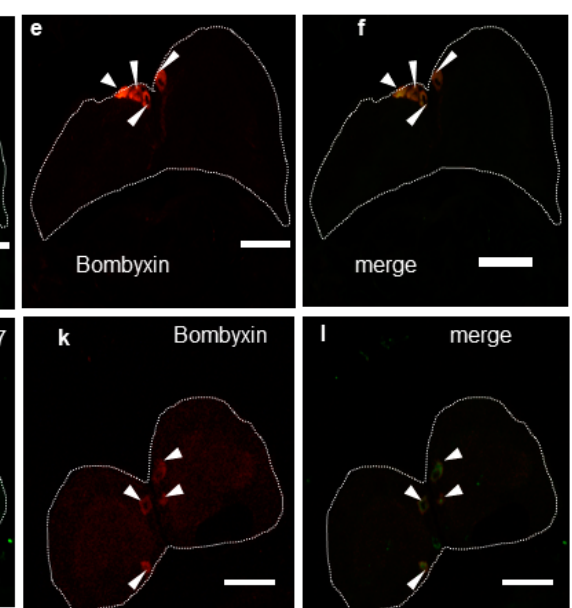

Fig. 2. Co-localization of Rab immunoreactivity (Rab1, 3, 6 and 7) and bombyxin immunoreactivity in the brain of Bombyx mori. Rab1 (a), 3 (d), 6 (g) and 7 (j) immunoreactivities were visualized using CF TM 488A (green fluorophore). Bombyxin immunoreactivity was visualized using CF TM 555 (red fluorophore) in the same images (b, e, h, k and n, respectively). Arrowheads indicate Rab-positive and bombyxin -positive cells. Rab3 (f) and Rab7 (I) -immunopositive cells were identical to bombyxin-immunopositive cells. Scale bar: 100 um. Control, $\mathrm{m}$ and $\mathrm{o}$. 

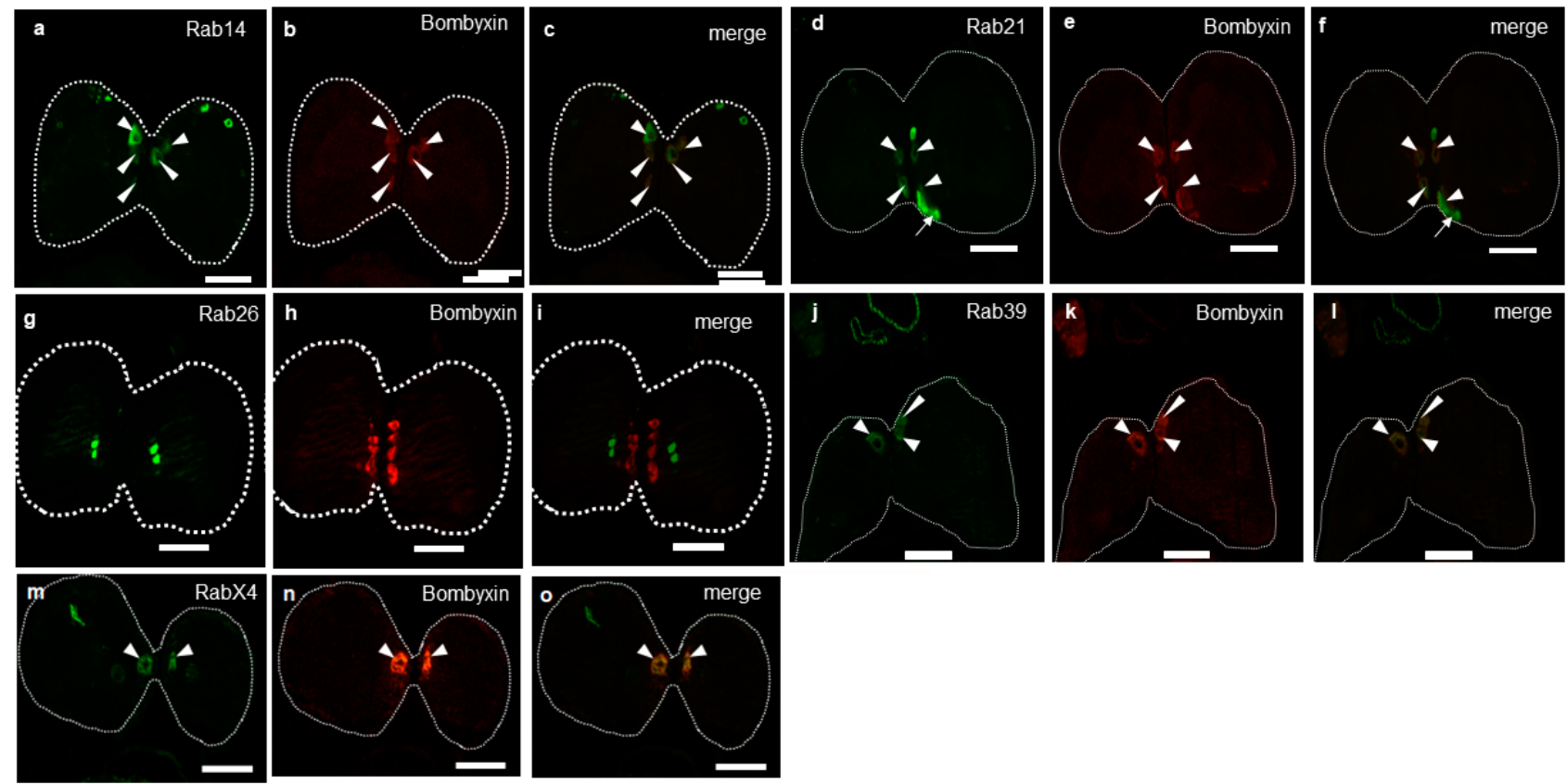

Fig. 3. Co-localization of Rab immunoreactivity (Rab14, 21, 26, 39 and X4) and bombyxin immunoreactivity in the brain of Bombyx mori. Rab14 (a), Rab21 (d), Rab26 (g) Rab39 (j)and RabX4 (m) immunoreactivities were visualized using CF ${ }^{\mathrm{TM}} 488 \mathrm{~A}$ (green fluorophore). Bombyxin immunoreactivity was visualized using CF ${ }^{\text {ТM }} 555$ (red fluorophore) in the same images (b, e, h, $k$ and $n$, respectively). Rab39 (I) and RabX4 (o) -immunopositive cells were identical to bombyxin-immunopositive cells. Rab14 and 21-immunopositive cells were partially identical to bombyxin-immunopositive cells ( $c$ and $f$, respectively; arrowheads). Rab26-immunopositive cells were not identical to bombyxin-immunopositive cells (i). Scale bar: $100 \mu \mathrm{m}$.

-RabX4 detected restricted areas in a set of neurons in the PI and dorsolateral protocerebrum (DP) (Figs 2 and 3). Anti-Rab26 detected a restricted area in a set of neurons in the DP (Fig. 3g).

\section{Rabs are present in the bombyxin-secretory neurons in the brain}

Anti-bombyxin detected a restricted set of neurons in the PI area (Fig. 2b). Double-labelling experiments showed that Rab1-, Rab3-, Rab6-, Rab7-, Rab14-, Rab21-, Rab39and RabX4-irs occurred in bombyxin-immunopositive neurons (Figs 2 and 3, arrowheads). Florescent images in Figs 2-3 show that only Rab3 and 39 occur exclusively in bombyxin cells in the brain (Fig. $2 \mathrm{f}$ and 31; arrowheads) and all other Rabs are found in additional "non-bombyxin" neurons. Rab26-ir did not occur in areas of bombyxin-ir (Fig. 3o).

\section{Rabs are present in the bombyxin-secretory neurons in axons of the CA}

Double-labelling experiments showed that Rab3-, Rab7-, Rab39- and RabX4-ir occurred in axonal projections of
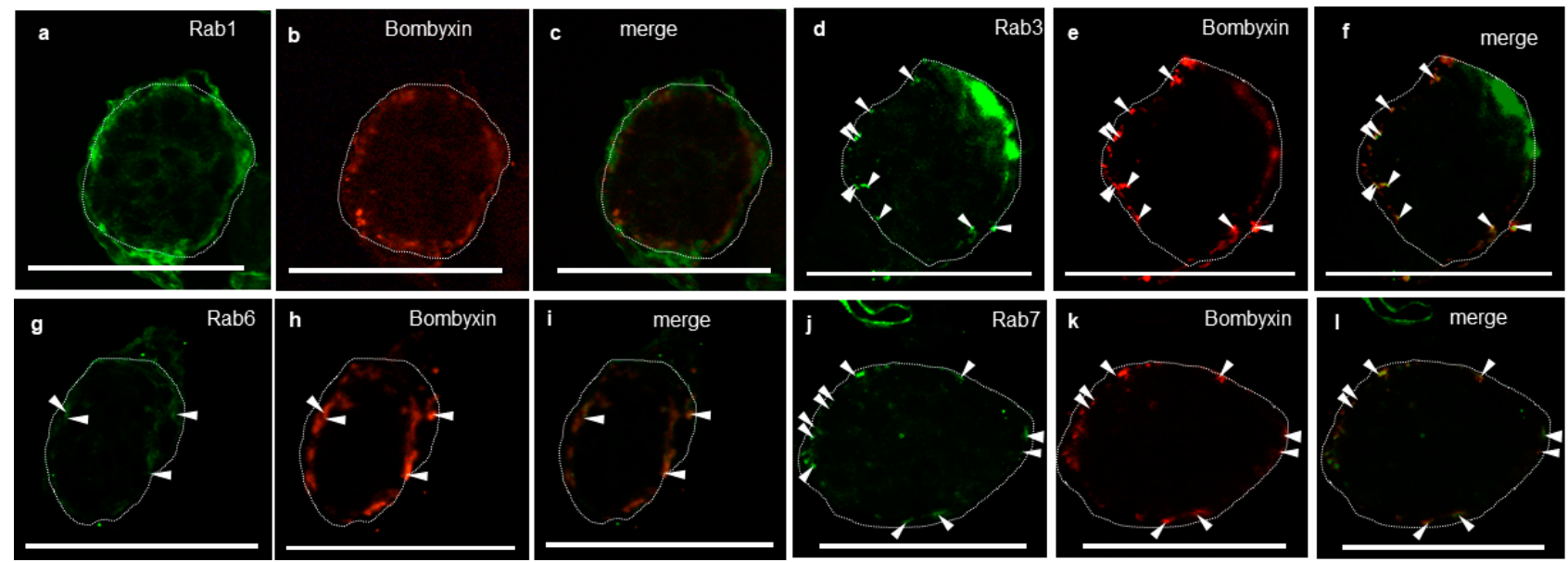

Fig. 4. Co-localization of Rab immunoreactivity (Rab1, 3, 6 and 7) and bombyxin immunoreactivity in the corpus allatum of Bombyx mori Rab1 (a), 3 (d), 6 (g) and 7 (j) immunoreactivities were visualized using CF ${ }^{\mathrm{TM}} 488 \mathrm{~A}$ (green fluorophore). Bombyxin immunoreactivity was visualized using $\mathrm{CF}^{\mathrm{TM}} 555$ (red fluorophore) in the same images (b, e, h and k, respectively). Rab3-and Rab7-immunopositive cells were almost identical to bombyxin-immunopositive cells ( $\mathrm{and} \mathrm{I}$, respectively; arrowheads). Rab1-immunopositive cells were not identical to bombyxin-immunopositive cells (c). Scale bar: $100 \mu \mathrm{m}$. 

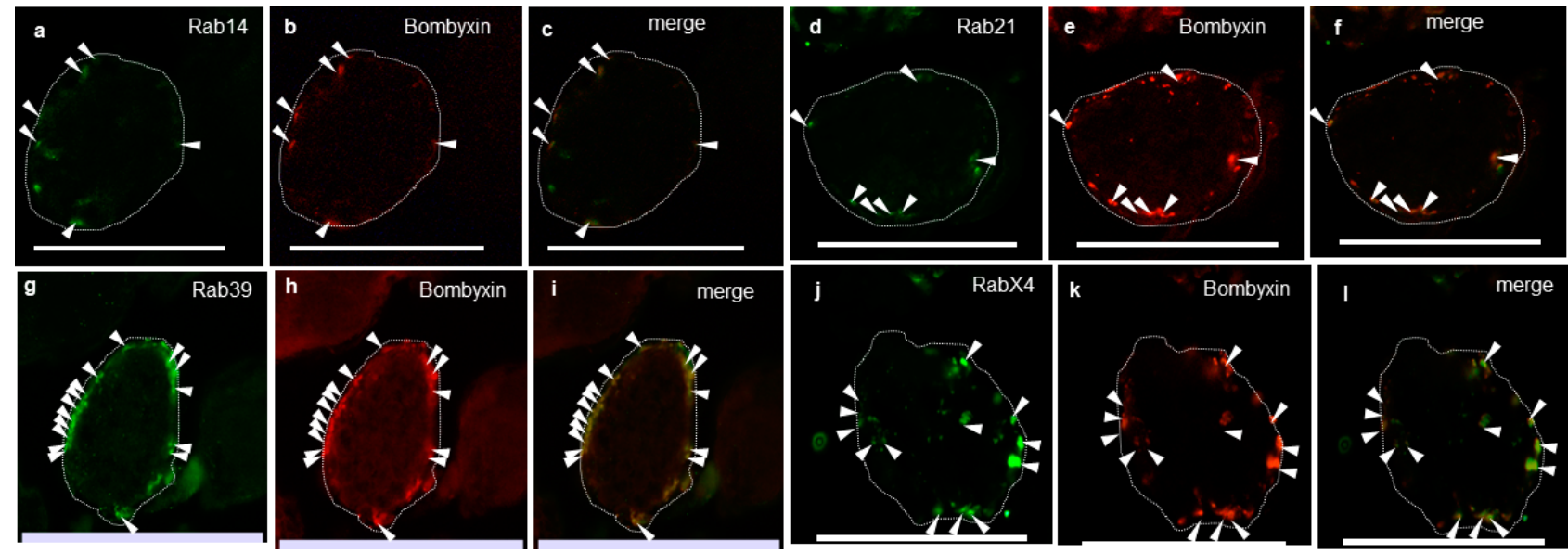

Fig. 5. Co-localization of Rab immunoreactivity (Rab14, 21, 39 and $X 4)$ and bombyxin immunoreactivity in the corpus allatum of Bombyx mori. Rab14 (a), 21 (d), 39 (g), X4 (j) immunoreactivities were visualized using CF ${ }^{\text {TM }} 488 \mathrm{~A}$ (green fluorophore). Bombyxin immunoreactivity was visualized using CF тм 555 (red fluorophore) in the same images ( $b, e, h$ and k, respectively). Rab39-and RabX4-immunopositive cells were almost identical to bombyxin-immunopositive cells (i and I, respectively; arrowheads). Rab14- and Rab21-immunopositive cells were partially identical to bombyxin-immunopositive cells (c and f, respectively; arrowheads). Scale bar: $100 \mu \mathrm{m}$.

bombyxin-immunopositive neurons in the CA (Figs 4 and 5). Using anti-Rab6, -Rab14 and -Rab21, immunohistochemistry detected only in a part of axonal projections of the bombyxin cells (Figs 4 and 5). The percentage of Rab-ir cells to bombyxin-immunopositive cells is shown in Fig. 6. The patterns of Rab3-, Rab7-, Rab39- and RabX4-ir cells are similar to that of bombyxin-ir cells (Figs 4f, 41, 5i and 51; arrowheads). More than $60 \%$ of Rab3-, Rab7-, Rab39- and RabX4-ir cells are bombyxin-ir cells (Fig. 6). Rab6-, Rab14- and Rab21-ir partially occurred in a part of bombyxin-immunopositive neurons (Figs 4i, 5c and 5f), but not Rab1-ir (Fig. 4c). Rab1 immunoreactivity was not detected in axons of the bombyxin-ir cells in CA, despite the fact that Rab1 was detected in cell bodies of bombyxin cells. These results indicate that Rab3, 7, 39 and $\mathrm{X} 4$ are involved in secretion of bombyxin from CA into the haemolymph.

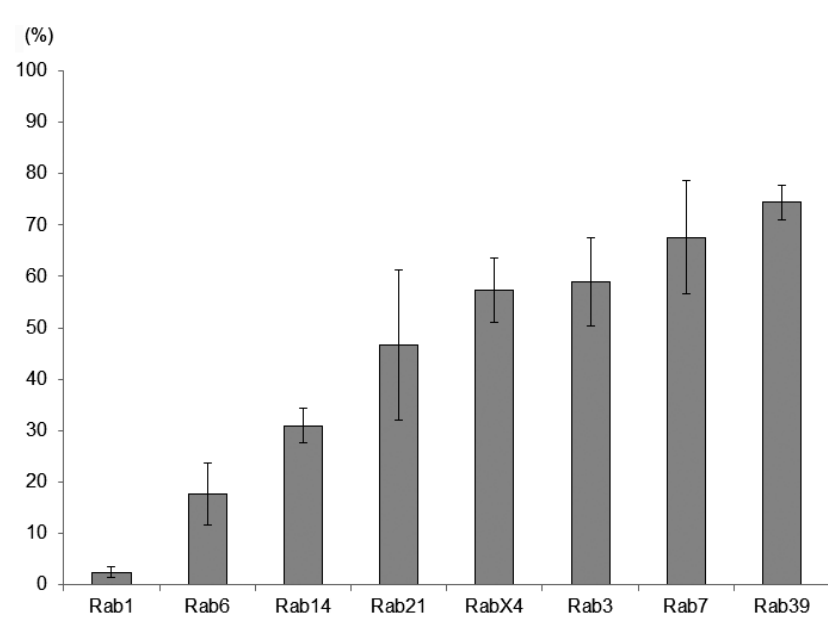

Fig. 6. The percentage of Rab-immunopositive cells that were stained by bombyxin-antibody in the corpus allatum of Bombyx mori. The $y$ axis is the percentage of cells identified by the Rab antibody that were stained with bombyxin antibody. Each value is the mean of three measurements. The statistical analysis was performed using the Tukey-Kramer method and Scheffe's F-test.

\section{Rab39-ir occurs in UNC-104-ir neurons in the brain and CA}

UNC104 is a kinesin-related protein (ATPase), the Drosophila ortholog of the kinesin-3 KIF1A, is a motor protein. UNC104 is required for ILPs transport of the insulinproducing cells in the brain of $D$. melanogaster (Cao et al., 2014). And Drosophila Rab39 interacts with UNC104. Double-labelling experiments showed that Rab39-ir occurred in UNC104-immunopositive neurons in the brain and in axons of CA (Fig. 7). Their presence suggests a possible role in the secretion of bombyxin.

\section{DISCUSSION}

Rab proteins are master mediators of vesicular membrane trafficking of endocytic and exocytic pathways, primarily recruiting proteins and lipids required for vesicle formation, docking and fusion (Pereira-Leal \& Seabra, 2001; Schwartz et al., 2007; Stenmark, 2009). Distinct Rab GTPases localize to different membrane compartments in order to determine the specificity and directionality of membrane trafficking pathways, mostly related to vesicular transport (Pylypenko et al., 2018).

Insulin and insulin-like peptides (ILPs) regulate numerous functions in insects including growth, development, carbohydrate metabolism and female reproduction. These neuropeptides are synthesized in specific neurosecretory cells, transported along the axons and secreted from the corpus allatum (CA) into the haemolymph. CA is a neuroendocrine organ together with the associated nerves, which synthesize juvenile hormone (Tobe \& Pratt, 1974). CA functions as a neurohemal organ for hormones synthesized in neurosecretory cells in the brain (Siga, 2003).

We tried to clarify the relationship between Rabs and bombyxin secretion using double-staining immunohistochemistry of the brain and CA of B. mori. Our results show that Rab3-ir, Rab7-ir, Rab39-ir and RabX4-irs were co-localized with bombyxin-ir in the axons of the CA. Of them, Rab39-ir fully overlapped with bombyxin-ir in the axons 

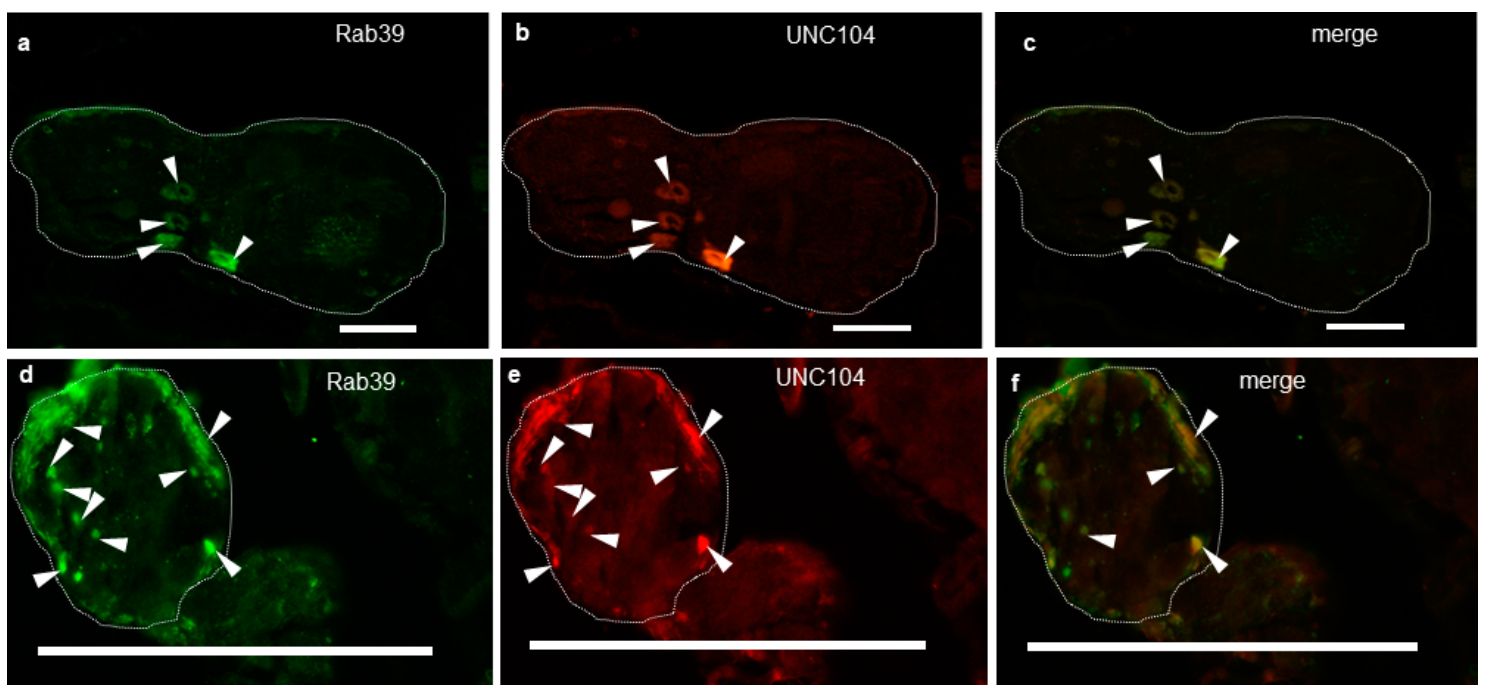

Fig. 7. Co-localization of Rab39 and UNC-104 immunoreactivities in the brain and corpus allatum of Bombyx mori. Rab39 immunoreactivity was visualized in the brain and corpus allatum using $\mathrm{CF}^{\mathrm{TM}} 488 \mathrm{~A}$ (green fluorophore; a, and d, respectively). UNC-104 immunoreactivity was visualized in the same tissues using CF ${ }^{\mathrm{TM}} 555$ (red fluorophore, b and e, respectively). Rab39 immunoreactivity occurred in UNC104immunopositive neurons (c and f). Scale bar: $100 \mu \mathrm{m}$.

of CA. This suggests that Rab39 mainly mediates the transport of bombyxin from the brain into the haemolymph.

The members of the Rab3 protein subfamily are the most abundant small GTPases in the brain. They are highly expressed in neurons where they specifically localize to synaptic vesicles and determine the efficiency of neurotransmitter release in neurons (Kittel \& Heckmann, 2016). Probably Rab3 mediates the release of bombyxin from the axons of CA directly into the haemolymph.

Rab7, a small GTPase of the Rab family associated with both the endosome and lysosome, has been investigated extensively and facilitates endosomal maturation, transport from the late endosome to the lysosome, and positioning of the endosome and lysosome via regulating their movement along cytoskeletons. In addition, Rab7 is involved in governing multiple trafficking processes including the biogenesis of the lysosome, phagosome, autophagosome and other lysosome-related organelles (Schwartz et al., 2007; Wang et al., 2011; Hyttinen et al., 2013). Rab7 may function in degradation of bombyxin in the brain and CA during starvation. Actually, there is an increase in the amount of Rab7 in the brain during starvation (data not shown).

D. melanogaster Rab X4 is enriched in the neuropil of neurons (Dunst et al., 2015) and exhibits a synapse-specific localization (Chan et al., 2011; Jin et al., 2012).

The use of $D$. melanogaster dominant-negative Rab constructs previously led to the identification of two Rab proteins (Rab5 and RabX4) involved in the internalization of rhodopsin and TRPL (the homolog of the transient receptor potential (trp)-like ion channel) from rhabdomeres to cells (Oberegelsbacher et al., 2011). RabX4, as well as Rab5, are essential for this process, which is related to endocytosis. Furthermore, D. melanogaster RabX4 did not co-localize with cysteine-string proteins, which are enriched in the synaptic vesicle, but it did co-localize with Rab11, which mediates the transport of recycling endosomes (Chan et al., 2011). These results suggest that RabX4 does not directly function in exocytosis, but is involved in the recovery of bombyxin from the haemolymph by the CA.

Rab39 is specifically expressed in the neuronal cells in the brain, as determined by qRT-PCR and in situ hybridization analyses (Giannandrea et al., 2010). In hippocampal neurons, Rab39 appears to localize to the Golgi complex as well as endosomes, and shRNA-mediated knockdown of Rab39 can reduce the number of growth cones at the neuronal terminal as well as synapse formation. In addition, Drosophila Rab39 interacts with UNC104, the Drosophila ortholog of the kinesin-3 KIF1A. UNC104 is required for neurite development and transporting insulin-like peptide along the axons of insulin producing cells. Rab39-ir occurred in the UNC104-ir in the brain and axons connecting the corpus cardiacum to the CA. Rab39 and UNC104 may mediate the transport of synaptic vesicle containing bombyxin from the brain to the CA.

Rab6-ir, Rab14-ir and Rab21-ir were localized in a part of axonal connections with bombyxin cells in the CA. Rab6 has been implicated in the function of intra-Golgi apparatus trafficking (Martinez et al., 1994) and exocytic transport to the plasma membrane (Grigoriev et al., 2007). Rab14 has been found to localize to the Golgi apparatus and rough ER compartments and to early endosome (Junutula et al., 2004) and is an important protein that mediates the interaction of phagosomes with early endocytic compartments and participates in the transport of endocytosing GLUT4 through early endosomes towards TGN (Reed et al., 2013; Okai et al., 2015). Rab6 and Rab14 may function in secretion of many neuropeptides containing bombyxin. Rab21 is located in early endosomes of human Hela cells (Simpson et al., 2004) and participates in endosomal trafficking of b1-integrins (Pellinen et al., 2008). As Rab21-ir was also detected in the other neurons of bombyxin-producing cells in the brain, Rab21 may function in the secretion of other neuropeptides. 
Rab1 proteins are involved in several cellular signalling pathways that include nutrient signalling (Thomas et al., 2014), Notch signalling (Charng et al., 2014), cell migration (Wang et al., 2010) and autophagy (Wang et al., 2015). Rab1-ir overlapped bombyxin-ir in the brain, but Rab1 was detected in cell bodies but not in the axons of bombyxin cells in the CA. Rab1 mediates ER to Golgi apparatus and intra-Golgi trafficking through different effectors. Rab1 may function in the transport from ER to Golgi apparatus in the brain, but does not function in the transport from the Golgi apparatus.

Rab26 did not overlap with bombyxin cells in the brain. In neurons, Rab26 is enriched in synaptic vesicles and adorns a subset of synaptic vesicles that have undergone recycling (Binotti et al., 2015). Overexpression of constitutively active Rab26 causes the appearance of large intracellular vesicular aggregates containing synaptic vesicle proteins such as synaptobrevin and Rab3. Rab26 may function in the transport of the other neuropeptides.

There are many Rab effectors that mediate the motility of organelles and vesicles; and contribute to the specificity of membrane traffic (Grosshans et al., 2006). Further studies are in progress to determine whether these effectors co-localize with Rab3, Rab7, RabX4 and Rab39 in the bombyxin producing cells in the brain and CA of $B$. mori.

ACKNOWLEDGEMENTS. We thank L. Benyon, of the Edanz Group (www.edanzediting.com/ac) for editing a draft of this manuscript. This work was supported by the Hyogo science and technology association.

\section{REFERENCES}

BARR F.A. 2013: Review series: Rab GTPases and membrane identity: causal or inconsequential? - J. Cell Biol. 202: 191199.

Binotti B., Pavlos N.J., Riedel D., Wenzel D., Vorbruggen G., Schalk A.M., Kuhnel K., Boyken J., Erck C., Martens H. et AL. 2015: The GTPase Rab26 links synaptic vesicles to the autophagy pathway. - eLife 4: e05597, 23 pp.

Brighouse A., DACKS J.B. \& Field M.C. 2010: Rab protein evolution and the history of the eukaryotic endomembrane system. - Cell. Mol. Life Sci. 67: 3449-3465.

Cao J., Ni J., Ma W., Shiu V., Milla L.A., Park S., Spletter M.L., TANG S., Zhang J., WeI X. et AL. 2014: Insight into insulin secretion from transcriptome and genetic analysis of insulin-producing cells of Drosophila. — Genetics 197: 175-192.

Caviglia S., Flores-Benitez D., Lattner J., Luschnig S. \& BrankatschK M. 2019: Rabs on the fly: Functions of Rab GTPases during development. - Small GTPases 10: 89-98.

Chan C.C., Scoggin S., Wang D., Cherry S., Dembo T., GreenBerg B., Jin E.J., Kuey C., Lopez A., Mehta S.Q. et AL. 2011: Systematic discovery of Rab GTPases with synaptic functions in Drosophila. - Curr. Biol. 21: 1704-1715.

Charng W.L., Yamamoto S., Jaiswal M., Bayat V., Xiong B., Zhang K., Sandoval H., David G., Gibbs S., Lu H.C. et al. 2014: Drosophila Tempura, a novel protein prenyltransferase alpha subunit, regulates notch signaling via Rab1 and Rab11. _ PLoS Biol. 12(1): e1001777, 16 pp.

Claeys I., Simonet G., Poels J., Van Loy T., Vercammen L., De Loof A. \& VANDEN BRoecK J. 2002: Insulin-related peptides and their conserved signal transduction pathway. - Peptides 23: 807-816.
Dunst S., Kazimiers T., von Zadow F., Jambor H., SAgner A., Brankatschk B., Mahmoud A., Spannl S., Tomancak P., Eaton S. ET AL. 2015: Endogenously tagged rab proteins: a resource to study membrane trafficking in Drosophila. - Dev. Cell 33: 351-365.

Elmogy M., Mohamed A.A., Tufail M., Uno T. \& Takeda M. 2018: Molecular and functional characterization of the American cockroach, Periplaneta americana, Rab5: the first exopterygotan low molecular weight ovarian GTPase during oogenesis. - Insect Sci. 25: 751-764.

Fujtta N., Huang W., Lin T.H., Groulx J.F., Jean S., Nguyen J., Kuchitsu Y., Koyama-Honda I., Mizushima N., Fukuda M. ET AL. 2017: Genetic screen in Drosophila muscle identifies autophagy-mediated T-tubule remodeling and a Rab2 role in autophagy. - eLife 6: e23367, 30 pp.

Garg A. \& Wu L.P. 2014: Drosophila Rab14 mediates phagocytosis in the immune response to Staphylococcus aureus. - Cell Microbiol. 16: 296-310.

Giannandrea M., Bianchi V., Mignogna M.L., Sirri A., Carrabino S., D’Elia E., Vecellio M., Russo S., Cogliati F., Larizza L. ET AL. 2010: Mutations in the small GTPase gene RAB39B are responsible for X-linked mental retardation associated with autism, epilepsy, and macrocephaly. - Am. J. Hum. Genet. 86: 185-195.

Gillingham A.K., Sinka R., Torres I.L., Lilley K.S. \& Munro S. 2014: Toward a comprehensive map of the effectors of Rab GTPases. - Dev. Cell 31: 358-373.

Grigoriev I., Splinter D., Keijzer N., Wulf P.S., Demmers J., Ohtsuka T., Modesti M., Maly I.V., Grosveld F., Hoogenraad C.C. ET AL. 2007: Rab6 regulates transport and targeting of exocytotic carriers. - Dev. Cell 13: 305-314.

Grosshans B.L., Ortiz D. \& Novick P. 2006: Rabs and their effectors: achieving specificity in membrane traffic. - Proc. Natl. Acad. Sci. USA 103: 11821-11827.

Heuer C.M., Kollmann M., Binzer M. \& Schachtner J. 2012: Neuropeptides in insect mushroom bodies. - Arthropod Struct. Dev. 41: 199-226.

Hyttinen J.M., NittTykoski M., Salminen A. \& KaARniranta K. 2013: Maturation of autophagosomes and endosomes: a key role for Rab7. - Biochim. Biophys. Acta 1833: 503-510.

Jin E.J., Chan C.C., Agi E., Cherry S., Hanacik E., Buszczak M. \& Hiesinger P.R. 2012: Similarities of Drosophila rab GTPases based on expression profiling: Completion and analysis of the rab-Gal4 kit. — PLoS ONE 7(7): e40912, 14 pp.

Junutula J.R., De Maziere A.M., Peden A.A., Ervin K.E., AdvaNI R.J., van DiJK S.M., Klumperman J. \& Scheller R.H. 2004: Rab14 is involved in membrane trafficking between the Golgi complex and endosomes. - Mol. Biol. Cell 15: 2218-2229.

Kittel R.J. \& Heckmann M. 2016: Synaptic vesicle proteins and active zone plasticity. — Front. Synaptic Neurosci. 8: 8, 8 pp.

Li G. \& MARLIN M.C. 2015: Rab family of GTPases. - Methods Mol. Biol. 1298: 16 pp.

Lighthouse D.V., Buszczak M. \& Spradling A.C. 2008: New components of the Drosophila fusome suggest it plays novel roles in signaling and transport. - Dev. Biol. 317: 59-71.

Martinez O., Schmidt A., Salamero J., Hoflack B., Roa M. \& Goud B. 1994: The small GTP-binding protein rab6 functions in intra-Golgi transport. - J. Cell Biol. 127: 1575-1588.

Mizoguchi A. \& Окамото N. 2013: Insulin-like and IGF-like peptides in the silkmoth Bombyx mori: discovery, structure, secretion, and function. - Front. Physiol. 4: 217, $11 \mathrm{pp}$.

Nagasawa H., Kataoka H., Isogai A., Tamura S., Suzuki A., Mizoguchi A., Fujiwara Y., TAKahashi S.Y. \& IshizaKi H. 1986: Amino acid sequence of a prothoracicotropic hormone of the 
silkworm Bombyx mori. — Proc. Natl. Acad. Sci. USA 83 5840-5843.

Nagata S., Hakuno F., Takahashi S. \& Nagasawa H. 2008: Identification of Bombyx mori Akt and its phosphorylation by bombyxin stimulation. - Comp. Biochem. Physiol. (B) 151: $355-360$

NASSEL D.R. 2002: Neuropeptides in the nervous system of Drosophila and other insects: multiple roles as neuromodulators and neurohormones. - Prog. Neurobiol. 68: 1-84.

Nassel D.R. \& VAnden BroecK J. 2016: Insulin/IGF signaling in Drosophila and other insects: factors that regulate production, release and post-release action of the insulin-like peptides. Cell Mol. Life Sci. 73: 271-290.

Nassel D.R., Liu Y. \& Luo J. 2015: Insulin/IGF signaling and its regulation in Drosophila. - Gen. Comp. Endocrinol. 221: 255-266.

Oberegelsbacher C., Schneidler C., Voolstra O., Cerny A. \& Huber A. 2011: The Drosophila TRPL ion channel shares a Rab-dependent translocation pathway with rhodopsin. - Eur. J. Cell Biol. 90: 620-630.

Okai B., Lyall N., Gow N.A., Bain J.M. \& Erwig L.P. 2015 Rab14 regulates maturation of macrophage phagosomes containing the fungal pathogen Candida albicans and outcome of the host-pathogen interaction. - Infect. Immun. 83: 1523 1535.

Pellinen T., Tuomi S., Arjonen A., Wolf M., Edgren H., Meyer H., Grosse R., Kitzing T., Rantala J.K., Kallioniemi O. et al. 2008: Integrin trafficking regulated by Rab21 is necessary for cytokinesis. - Dev. Cell 15: 371-385.

Pereira-Leal J.B. \& Seabra M.C. 2001: Evolution of the Rab family of small GTP-binding proteins. - J. Mol. Biol. 313: 889-901.

Pfeffer S.R. 2013: Rab GTPase regulation of membrane identity. - Curr. Opin. Cell Biol. 25: 414-419.

PfEFfer S.R. 2017: Rab GTPases: master regulators that establish the secretory and endocytic pathways. - Mol. Biol. Cell 28: $712-715$.

Pylypenko O., Hammich H., Yu I.M. \& Houdusse A. 2018: Rab GTPases and their interacting protein partners: Structural insights into Rab functional diversity. - Small GTPases 9: $22-48$.

Reed S.E., Hodgson L.R., Song S., May M.T., Kelly E.E., McCaffrey M.W., Mastick C.C., Verkade P. \& Tavare J.M. 2013: A role for Rab14 in the endocytic trafficking of GLUT4 in 3T3-L1 adipocytes. - J. Cell Sci. 126: 1931-1941.

Satake S., Masumura M., Ishizaki H., Nagata K., Kataoka H., Suzuki A. \& Mizoguchi A. 1997: Bombyxin, an insulin-related peptide of insects, reduces the major storage carbohydrates in the silkworm Bombyx mori. - Comp. Biochem. Physiol. (B) 118: $349-357$.

Schoofs L., De Loof A. \& VAn Hiel M.B. 2017: Neuropeptides as regulators of behavior in insects. - Annu. Rev. Entomol. 62: $35-52$.

Schwartz S.L., Cao C., Pylypenko O., Rak A. \& WandingerNess A. 2007: Rab GTPases at a glance. - J. Cell Sci. 120: 3905-3910.

SigA S. 2003: Anatomy and functions of brain neurosecretory cells in Diptera. - Microsc. Res. Tech. 62: 114-131.

Simpson J.C., Griffiths G., Wessling-Resnick M., Fransen J.A., BENNETT H. \& JoNES A.T. 2004: A role for the small GTPase Rab21 in the early endocytic pathway. - J. Cell Sci. 117: 6297-6311.

STEnMARK H. 2009: Rab GTPases as coordinators of vesicle traffic. - Nat. Rev. Mol. Cell Biol. 10: 513-525.
Sugawara T., Kano F. \& Murata M. 2014: Rab2A is a pivotal switch protein that promotes either secretion or ER-associated degradation of (pro)insulin in insulin-secreting cells. - Sci. Rep. 4: 6952, 14 pp.

Thomas J.D., Zhang Y.J., Wei Y.H., Cho J.H., Morris L.E., Wang H.Y. \& ZHENG X.F. 2014: Rab1A is an mTORC1 activator and a colorectal oncogene. - Cancer Cell 26: 754-769.

Tobe S.S. \& Pratt G.E. 1974: Dependence of juvenile hormone release from corpus allatum on intraglandular content. $-\mathrm{Na}$ ture 252: 474-476.

Uno T., Nakada T., Okamaoto S., Nakamura M., Matsubara M., Imaishi H., Yamagata H., Kanamaru K. \& Takagi M. 2007: Determination of phosphorylated amino acid residues of Rab8 from Bombyx mori. - Arch. Insect Biochem. Physiol. 66: 89-97.

Uno T., Sakamoto K., Isoyama Y., Hiragaki S., Uno Y., Kanamaru K., Yamagata H., Takagi M., Mizoguchi A. \& Takeda M. 2013: Relationship between the expression of Rab family GTPases and neuropeptide hormones in the brain of Bombyx mori. - Histochem. Cell Biol. 139: 299-308.

Uno T., Isoyama Y., Sakamoto K., Uno Y., Sakamoto K., Kanamaru K., Yamagata H., Takagi M., Mizoguchi A. \& Takeda M. 2014: Characterization of Rab-interacting lysosomal protein in the brain of Bombyx mori. - Histochem. Cell Biol. 141: 311-320.

Uno T., Furutani M., Watanabe C., Sakamoto K., Uno Y., Kanamaru K., Yamagata H., Mizoguchi A. \& Takeda M. 2016: Rab proteins in the brain and corpus allatum of Bombyx mori. - Histochem. Cell Biol. 146: 59-69.

Uno T., Ozakiya Y., Furutani M., SaKamoto K., Uno Y., KaJIwara H., Kanamaru K. \& Mizoguchi A. 2019: Functional characterization of insect-specific RabX6 of Bombyx mori. - Histochem. Cell Biol. 151: 187-198.

Uytterhoeven V., Kuenen S., Kasprowicz J., Miskiewicz K. \& VERSTREKEN P. 2011: Loss of skywalker reveals synaptic endosomes as sorting stations for synaptic vesicle proteins. Cell 145: 117-132.

Van de Velde S., Badisco L., Claeys I., Verleyen P., Chen X., Vanden Bosch L., Vanden Broeck J. \& Smagghe G. 2007: Insulin-like peptides in Spodoptera littoralis (Lepidoptera): Detection, localization and identification. - Gen. Comp. Endocrinol. 153: 72-79.

WANG Z. \& ThURMOND D.C. 2009: Mechanisms of biphasic insulin-granule exocytosis - roles of the cytoskeleton, small GTPases and SNARE proteins. - J. Cell Sci. 122: 893-903.

Wang C., Yoo Y., Fan H., Kim E., Guan K.L. \& Guan J.L. 2010: Regulation of Integrin beta 1 recycling to lipid rafts by Rab1a to promote cell migration. - J. Biol. Chem. 285: 29398-29405.

Wang T., Ming Z., Xiaochun W. \& Hong W. 2011: Rab7: role of its protein interaction cascades in endo-lysosomal traffic. Cell Signal. 23: 516-521.

Wang J., Davis S., Menon S., Zhang J., Ding J., Cervantes S., Miller E., Jiang Y. \& Ferro-Novick S. 2015: Ypt1/Rab1 regulates Hrr25/CK1delta kinase activity in ER-Golgi traffic and macroautophagy. - J. Cell Biol. 210: 273-285.

White M.F. 2002: IRS proteins and the common path to diabetes. - Am. J. Physiol. Endocrinol. Metab. 283: E413-422.

Ye T., TANG W. \& Zhang X. 2012: Involvement of Rab6 in the regulation of phagocytosis against virus infection in invertebrates. - J. Proteome Res. 11: 4834-4846.

Received April 11, 2021; revised and accepted September 14, 2021 Published online October 7, 2021 